\title{
Besonderheiten eines Bandscheibenvorfalles bei Spondylolisthese
}

\author{
J. Grifka, J. Möller \\ Orthopädische Universitätsklinik im St. Josef-Hospital, Gudrunstr. 56, 4630 Bochum (Direktor: Prof. Dr. J. \\ Krämer)
}

\section{Zusammenfassung}

Die Behandlung von Bandscheibenvorfällen bei Spondylolisthesen unterscheidet sich bezüglich OP-Indikation und operativen Verfahren prinzipiell nicht von dem sonst üblichen Vorgehen, sofern die Ischialgie nicht durch das retrolisthetische Füllgewebe oder die Wirbelkörperkante provoziert ist. Im vorliegenden Fall eines Bandscheibenvorfalles L5/S1 bei Olisthese Grad I mit typischem L5-Syndrom wurde das Prolaps-Gewebe und der angrenzende Teil des retrolisthetischen Füllgewebes in üblicher MikrodiskotomieTechnik entfernt. Eine unmittelbar postoperativ verstärkte L5-Symptomatik zwang zur operativen Reintervention, wobei sich ein Dearrangement des retrolisthetischen Füllgewebes als Ursache hierfür fand.

\section{Unexpected Results in a Case of Disc Herniation with Spondylolisthesis}

Treatment of intervertebral disc herniation associated with spondylolisthesis is not different from common procedures concerning indication for surgery and surgical technique as far as sciatica is not related to retrolisthetic soft tissue or the posterior edge of the vertebral body. In a case of a disc herniation L5/ $\mathrm{S} 1$ and an olisthesis grade I with radicular pain L5 a microdiscectomy of the prolapse and parts of the retrolisthetic soft tissue was performed. An immediate reintervention was necessary due to postoperative symptoms of paralysis. Dearrangement of the retrolisthetic soft tissue was found to cause the increased L5symptoms.

Spondylolisthesen und Spondylolysen zählen zu den prädiskotischen Deformitäten (Krämer, 1986). Durch den Gleitvorgang wird die betroffene Bandscheibe zermürbt und ausgewalzt, so daß es in der Folge zu Bandscheibenvorfällen und einer weiteren Lockerung kommen kann.

Liegen außerdem Assimilationsstörungen am lumbosakralen Übergang vor, so ist die Rate der Bandscheibenvorfälle bereits aufgrund dessen deutlich erhöht (Lange, 1965; Wansor u. Fleischhauer, 1986). Eine hypersegmentierte LWS ist wesentlich mobiler und bedarf zur Stabilisierung eines guten Muskelkorsetts (Wansor $\mathrm{u}$. Fleischhauer, 1986).

Z. Orthop. 129 (1991) 362-364

(c) 1991 F. Enke Verlag Stuttgart
Eine Ischialgie bei Spondylolisthese ist meist doppelseitig. Hierfür werden fibröse Wucherungen verantwortlich gemacht, die im Bereich der Bogenspalte und als Füllgewebe im dorsalen Bereich des Gleitwirbels vorhanden sind. Die Spannung der Nervenwurzeln über dieses Füllgewebe und die Hinteroberkante des unter dem Gleitwirbel befindlichen Wirbels kann zu Nervenwurzelirritation beim Wirbelgleiten führen (Krämer, 1986).

Der vorliegende Fall zeigt die beondere Problematik des intraoperativen Befundes anläßlich einer Diskotomie, mit nachfolgender postoperativer Pareseverstärkung, die eine Revision erforderlich machte.

\section{Ausgangsbefunde}

Bei dem 42-jährigen, athletischen Patienten bestand als Grundleiden eine Spondylolisthese L5/S1, Meyerding Grad I, bei Lumbalisation von S1 mit Ausbildung von Assimilationsgelenken (Abb. 1). In der Vorgeschichte bestanden seit einigen Jahren rezidivierende Beschwerden im Rückenbereich mit wechselnder Ausstrahlung in die Beine. Nach dem Skisport ist es zu einer Exacerbation der Beschwerden im rechten Bein gekommen. Klinisch fand sich eine mäßiggradige Verspannung der Rückenstreckmuskulatur mit einer Einschränkung der Rumpfvorbeugung. Die Schmerzausstrahlung entsprach dem Dermatom L5. Während Zehenspitzenstand und -gang regelrecht auszuführen waren, war der Fersenstand und -gang rechtsseitig nur mit deutlich abgeschwächter Kraft möglich. Fußheber und Großzehenheber zeigten im Seitenvergleich rechtsseitig eine deutliche Kraftminderung, während Fußrandheber, Kniestrecker und Fußsenker seitengleich kraftvoll angespannt werden konnten. Der Lasègue lag rechts bei $60^{\circ}$. Reflexauffälligkeiten zeigten sich nicht. Auch der positive Hyperextensionstest läßt keine Gewichtung in der Hinsicht zu, daß die Schmerzverstärkung der Olisthese zugeordnet werden könnte.

Eine aktuelle Kernspintomographie zeigte eine mäßige Vorwölbung der Bandscheibe L5/S1 bei einem geräumigen lumbalen Spinalkanal (Abb. 2). Die austretende rechte L5-Wurzeltasche war nach dorsal abgedrängt und beengt. Die klinische Symptomatik des Patienten war somit durch den Bandscheibenvorfall L5/S1 zu erklären. Bending-Aufnahmen der Wirbelsäule waren aufgrund der akuten radikulären Schmerzsymptomatik nicht mit weiterführender diagnostischer Aussage durchzuführen. Es wurde die Indikation zur Diskotomie in Mikrotechnik gestellt. Eine Fusion war angesichts der erstgradigen Olisthese mit seitenbetonter radikulärer Symptomatik primär nicht in Erwägung zu ziehen, wie von Gill (1955) belegt. 


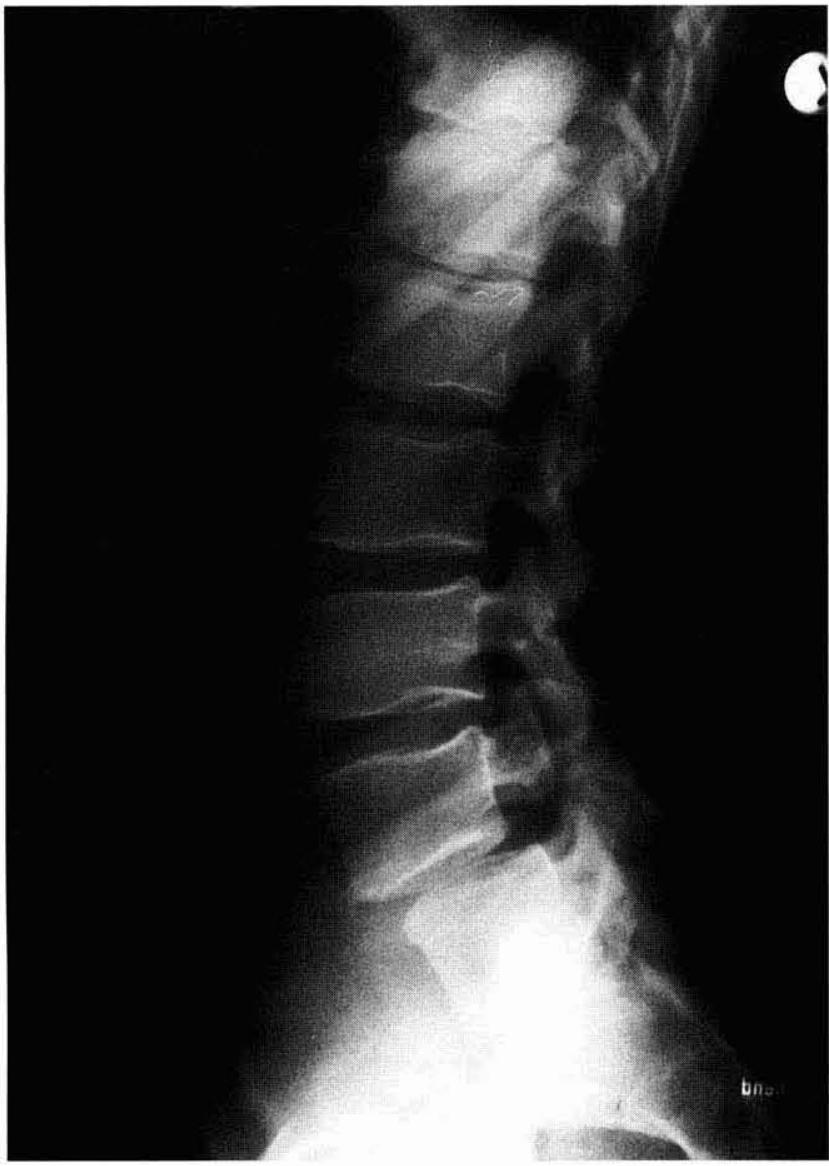

Abb. 1 Spondylolisthese L5/S1, Grad Meyerding I, Lumbalisation von $\mathrm{S} 1$

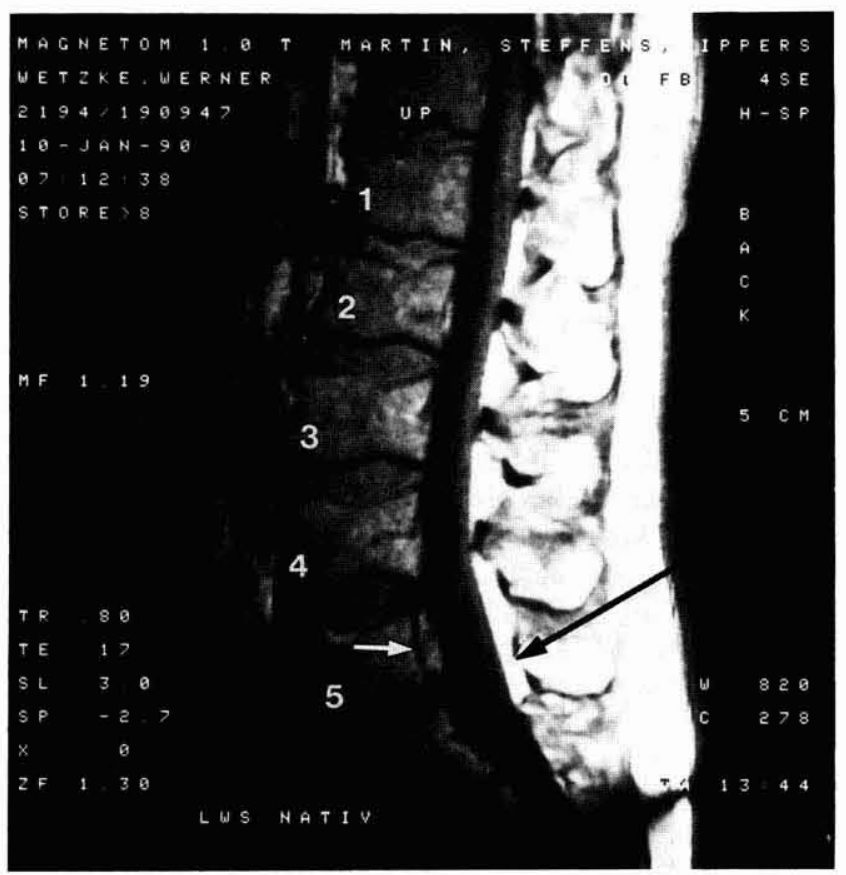

Abb. 2 Präoperatives Kernspintomogramm: Mäßige Vorwölbung der Bandscheibe L5/S1, spondylolisthetisches Füllgewebe dorsal LWK V

\section{OP-Vorgehen}

Das mikrochirurgische Operationsverfahren bei Bandscheibenvorfall erlaubt bei minimalem OPTrauma (Krämer, 1990) das exakte Zugehen auf den auffälligen Befund. So wurde in Knie-Hocklagerung mit druckfreier Position des Abdomens in typischer Weise eine partielle Flavektomie L5/S1 rechts durchgeführt, Prolaps- und retrolisthetisches Füllgewebe extrahiert, das die Wurzel L5 bedrängte, und bei spannungsfreiem Verlauf der Nervenwurzel die Sondierung zum Foramen intervertebrale sowie nach kaudal und kranial im Spinalkanal vorgenommen. Das Vorgehen unterschied sich in keiner Weise von dem sonst üblichen bei Bandscheibenprolaps.

\section{Postoperativer Befund}

Unmittelbar postoperativ bestand klinisch eine an Paralyse grenzende Fußheberparese und Großzehenparese rechts. Bei einer neurologischen Konsiliaruntersuchung zeigte sich im EMG keine Denervierung der Fußhebermuskulatur, was auf eine relativ frische Läsion hindeutet. Die Peroneus-SEP wiesen auf eine wirbelsäulennahe Schädigung hin.

Vom intraoperativen Befund her war diese Problematik unerklärlich. Ein differentialdiagnostisch zu erwägendes postoperatives Ödem schied wegen der unmittelbar postoperativ eingetretenen Verstärkung der Parese aus.

Eine Computertomographie-Kontrolle zeigte eine weichteildichte Raumforderung dorsal der rechten Wirbelkörperkante von L5, die als partiell verkalkter Bandscheibenprolaps gewertet wurde (Abb. 3).

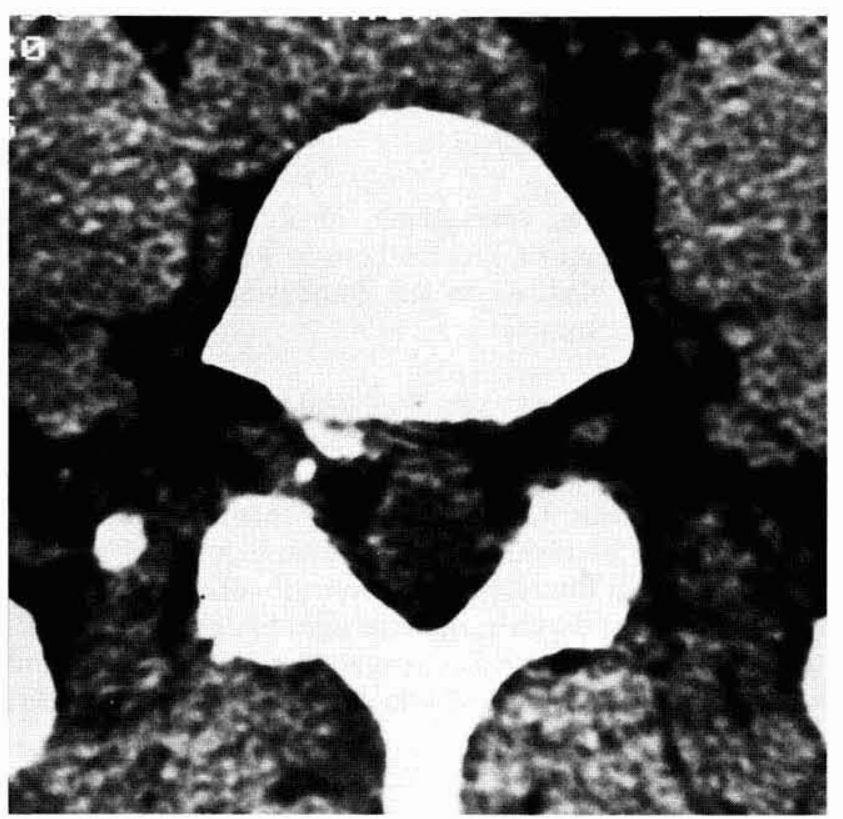

Abb. 3 Postoperatives Computertomogramm: Weichteildichte Raumforderung LWK V rechts mediolateral; beim Revisionseingriff als dislozierte, verhärtete Anteile des retrolisthetischen Füllgewebes identifiziert. 


\section{Operative Revision}

Die unmittelbare operative Revision zeigte keinerlei Anhaltspunkte für eine Infektion, ein Hämatom oder andere Kompressionsursachen. Es wurde eine erweiterte Dekompression der Wurzel L5 rechts durch Abtragung des Bogens L5 vorgenommen, sowie die Entfernung des Pseudarthrosengewebes. Dabei wurden außerdem noch verhärtetes, zum Teil sklerosiertes Füllgewebe und weitere Anteile sequestrierten Bandscheibengewebes entfernt.

\section{Schlußfolgerungen und weitere Maßnahmen}

Retrospektiv muß davon ausgegangen werden, daß durch ein Dearrangement des spondylolisthetischen Füllgewebes nach Bandscheibenoperation eine vermehrte Wurzelkompression eingetreten ist, die bei der ersten Operation in der Knie-Hocklage bei dem üblichen Sondieren des Wurzelabganges und des Spinalkanales, der ein relativ weites Lumen hatte, nicht auffällig war.

Zur Reaktivierung der Peronealgruppe wurden entsprechende krankengymnastische und physikalische Maßnahmen durchgeführt. Aufgrund des Lokalbefundes einer in einem Kernspintomogramm nach dem zweiten operativen Eingriff relativ gut wasserhaltig erscheinenden Bandscheibe L5/S1 wurde von einer stabilisierenden Fusionsoperation derzeit abgesehen. Bei einer Nachuntersuchung 6 Wochen postoperativ zeigte sich eine restitutio ad integrum, ohne muskuläre Restschwäche. Seit Ablauf der 12. postoperativen Woche geht der Patient seiner vorherigen sportlichen und beruflichen Belastung als Bergsteiger und Sportlehrer nach.

Dieser Fall mahnt zur möglichst weitgehenden Entfernung des spondylolisthetischen Füllgewebes, da sonst die Gefahr besteht, daß ein sekundäres postoperatives Dearrangement zur Nervenwurzelkompression führt. Die Indikation zur Bandscheibenoperation bei Spondylolisthese weicht nicht von den sonst üblichen Richtlinien $\mathrm{ab}$.

\section{Literatur}

Gill, G. G., J. G. Manning, H. L. White: Surgical Treatment of Spondylolisthesis without Spine Fusion. J. Bone Joint Surg. 37-A (1955) 493520

Krämer, J.: Bandscheibenbedingte Erkrankungen. 2. Aufl. Thieme, Stuttgart 1986

Krämer, J.: Die mikrochirurgische Operation beim lumbalen Bandscheibenvorfall. Operat. Orthop. Traumatol. 2 (1990), 84-92

Lange, M.: Lehrbuch der Orthopädie und Traumatologie. Enke, Stuttgart 1965

Wansor, S., I. Fleischhauer: Röntgenbefunde an der Brust- und Lendenwirbelsäule bei Frauen und Männern mit und ohne Rückenbeschwerden. Med. Diss., Bochum 1986

\section{Dr. med. J. Grifka}

Orthopädische Universitätsklinik

im St. Josef-Hospital

Gudrunstr. 56

4630 Bochum 